\title{
QGP tomography with photon tagged jets in ALICE*
}

\author{
Yaxian $\mathrm{Mao}^{1,3}$, Yves Schutz ${ }^{2}$, Daicui Zhou ${ }^{1}$, Christophe Furget ${ }^{3}$, Gustavo Conesa Balbastre ${ }^{4}$ \\ 1 (Institute of Particle Physics, Huazhong Normal University, Wuhan 430079, China ) \\ 2 (CERN, Geneva 23, Switzerland) \\ 3 (Laboratoire de Physique Subatomique et de Cosmologie, CNRS/IN2P3, Grenoble 38026, France) \\ 4 (Laboratori Nazionali di Frascati, INFN, Frascati, Italy)
}

\begin{abstract}
$\gamma+$ jet events provide a tomographic measurement of the medium formed in heavy ion collisions at LHC energies. Tagging events with a well identified high $p_{T}$ direct photon and measuring the correlation distribution of hadrons emitted oppositely to the photon, allows us to determine, with a good approximation, both the jet fragmentation function and the back-to-back azimuthal misalignement of the direct photon and the jet. Comparing these two observables measured in $p p$ collisions with the ones measured in $A A$ collisions will reveal the modifications of the jet structure induced by the medium formed in $A A$ collisions and consequently will infer the medium properties.
\end{abstract}

Key words direct photon, QGP, jet structure, tomography, path length

PACS 24.85.+p, 25.75.Bh, 25.75. Cj, 25.75.Nq

\section{Introduction}

The Large Hadron Collider (LHC) at CERN, will collide heavyions at unprecedented high energies, exceeding by a factor 30 the energy available at RHIC 1 . The main objective of ALICE (A Large Ion Collider Experiment) ${ }^{2]}$, is to study matter under extreme conditions of energy density to gain a better understanding of the fundamental properties of the strong interaction. In particular, ALICE will explore the Quark-Gluon Plasma (QGP), the state of deconfined matter predicted by $\mathrm{QCD}^{3}$. The medium formed in heavy-ion collisions can be best probed by hard scattered partons produced in $2 \rightarrow 2$ QCD processes at the leading order (LO) including in the final state a hard direct photon (Compton scattering: $q$ $+\mathrm{g} \rightarrow \gamma+\mathrm{q}$ and quark annihilation: $\mathrm{q}+\bar{q} \rightarrow \gamma+\mathrm{g})$. On one hand, the 4-momentum of the scattered parton is modified while traversing the medium, and on the other hand, the scattered photon does not interact, thus providing a reference for the 4-momentum of the partner parton. Hence, from the modification experienced by the hard scattered partons, measured though photon tagged jets, the medium properties can be inferred. In particular, since these hard scattering processes sample the entire collision volume, the final state hadronic observables provide a real tomographic probe of the medium 4 .

Several algorithms ${ }^{5}$ have been developed to identify $\gamma$-jet events in $\mathrm{p}-\mathrm{p}$ and $\mathrm{Pb}-\mathrm{Pb}$ collisions, demonstrating the feasibility of such measurements with the ALICE detectors. However, the jet identification remains challenging in the heavy-ion environment in particular for the energies $E_{\gamma} \sim 30 \mathrm{GeV}$ where $\gamma$-jet events are measurable in ALICE with sufficient statistics. An equivalent approach is to measure directphoton-hadrons correlation ${ }^{6}$.

In the following, we have first established the intrinsic properties $\left(k_{T}\right)$ of $\gamma$-jet events expected in pp collisions at LHC energies. Then we discuss the nucleus-nucleus (AA) collision case, in particular, we explore the possibility to select $\gamma$-jet events as a function of their localization in the medium to validate the tomographic approach.

Received 25 November 2009

* This work is supported partly by the NSFC (10875051 and 10635020), the State Key Development Program of Basic Research of China (2008CB317106), the Key Project of Chinese Ministry of Education (306022 and IRT0624) and the Programme of Introducing Talents of Discipline to Universitiesnder of China: B08033

1) E-mail: maoyx@iopp.ccnu.edu.cn

(C) 2009 Chinese Physical Society and the Institute of High Energy Physics of the Chinese Academy of Sciences and the Institute of Modern Physics of the Chinese Academy of Sciences and IOP Publishing Ltd 


\section{$2 \gamma$-hadron topololigy in pp collisions}

At leading order perturbative QCD, a pair of hard-scattered partons emerges exactly back-to-back in the center of mass of the partonic system. Due to the finite size of the proton, however, it was found that each of the colliding parton carries initial transverse momentum with respect to the colliding axis, originally described as "intrinsic $k_{T}$ ". Beyond the leading order, initial and final state radiations (ISR/FSR) will generate additional transverse momentum. Therefore, the resulting total transverse momentum of the outgoing parton pair causes an acoplanar and a momentum imbalance, $\left\langle k_{T}>\mathbf{7}\right.$. It is measured as the net transverse momentum of the outgoing parton-pair $\left\langle p_{T}\right\rangle_{\text {pair }}=\sqrt{2} \cdot\left\langle k_{T}\right\rangle$. It is anticipated that medium effects will generate additional transverse momentum resulting in a broadening of $k_{T}$. This transverse momentum broadening can be directly related to the transport parameter $\hat{q}$, which describes the transverse momentum transferred from the medium to the traversing parton ${ }^{8}$.

Using the PYTHIA event generator ${ }^{9}$, we have established the collision energy dependence of $\left\langle k_{T}\right\rangle$ from $\gamma$-jet events, by taking available data from different experiments measurements 10 and extrapolate to the LHC energies. The dependence is < $p_{T}>_{\text {pair }}=A \cdot \log (B \cdot \sqrt{s})$ with $A=2.064 \pm 0.171$ and $B=0.164 \pm 0.045$.

To study the dependence of $<k_{T}>$ with the transverse momentum of the hard scattering, we have generated $\gamma$-jet and jet-jet events with PYTHIA generator in different $p_{T}$ bins with collision energy $14 \mathrm{TeV}$, within $k_{T}$ setting predicted above and ISR/FSR on. The averaged $\left\langle p_{T}\right\rangle_{\text {pair }}$ versus the transverse momentum, shows a weakly linear dependence.

\section{Medium modification by heavy ion collisions}

The tomography measurement can be performed by selecting $\gamma$-h pairs with different values of the parameter $x_{E}=-\vec{p}_{T}^{h} \cdot \vec{p}_{T}^{\gamma} /\left|p_{T}^{\gamma}\right|^{2}$. This criteria can effectively control hadron emission from different regions of the medium and therefore extract the corresponding jet modification parameters 4 .

To simulate the medium induced energy loss, we used the Monte-Carlo model QPYTHIA 11, which combines an energy loss mechanism $\stackrel{12}{12}$ and a realistic description of the collision geometry $\stackrel{[13}{~}$. The HIJING 14 generator was used to simulate the underlying events of heavy-ion collisions and PYTHIA to simulate pp collisions. Three samples of $\gamma$-jet events were generated with photon energy larger than $20 \mathrm{GeV}$. A first sample of pp collisions at $5.5 \mathrm{TeV}$ generated with PYTHIA provides the baseline. The second sample consists in similar events modified by QPYTHIA merged with central collision events . The last sample is obtained by merging the PYTHIA events and peripheral collision events.

Tagging events with a direct photon well identified ${ }^{[15}$ by the ALICE calorimeters and measuring the distribution of hadrons emitted oppositely to the photon as a function of $x_{E}$, allows us to determinate the jet fragmentation function ${ }^{6}$. The underlying event is subtracted by correlating the isolated photon with charged hadrons emitted on the same side as the photon, in the azimuthal range $-\pi / 2<\Delta \phi<\pi / 2$. To quantify the medium modification, $I_{A A}$ is calculated (Fig. 1)

$$
I_{A A}\left(x_{E}\right)=\frac{C F_{A A}}{C F_{p p}}
$$

as the ratio of $\gamma$-hadrons correlation distribution measured in AA and pp collisions. The expected enhancement at low $x_{E}$ and suppression at high $x_{E}$ for central collision is observed, whereas, $I_{A A}$ is equal to 1 for peripheral collisions, where quenching effects are absent.

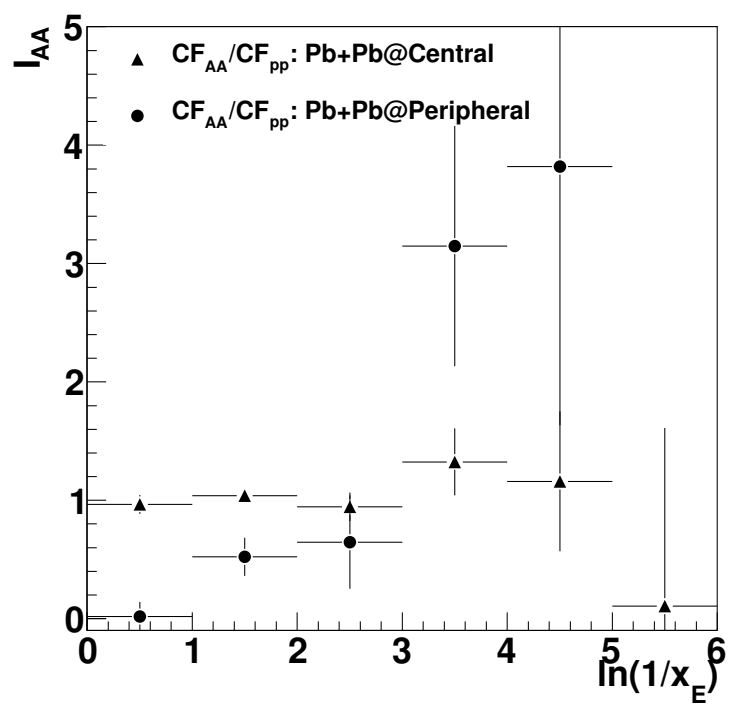

Fig. 1. The nuclear modification factor $I_{A A}$ for $\gamma$-hadrons correlation distribution in 
central and peripheral $\mathrm{Pb}+\mathrm{Pb}$ collisions at $\sqrt{s_{N N}}=5.5 \mathrm{TeV}$.

To illustrate the selectivity of the tomographic measurement, the length, L, the jet travels inside the medium is calculated. Fig. 2 indicates that most high $p_{T}$ leading particles are preferentially produced at the surface (small L), while low $p_{T}$ leading particles are produced inside the whole volume (large L), which demonstrates the $\mathrm{L}$ dependence of of the $\gamma$ tagged charged hadron production for 2 different $x_{E}$ regions.

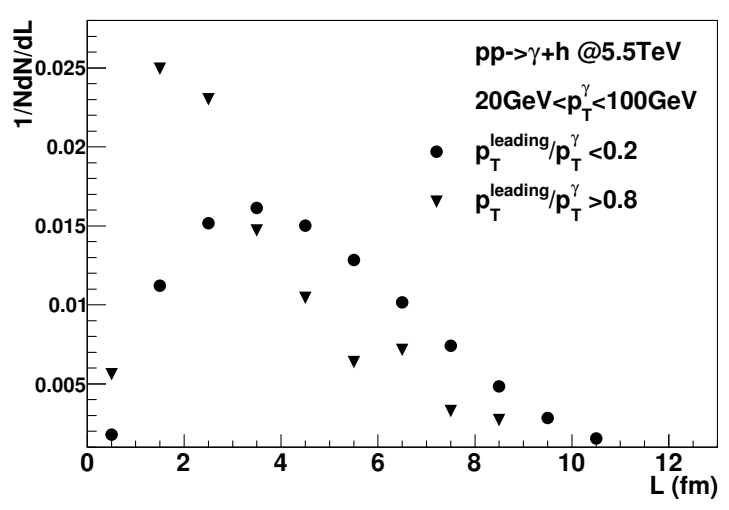

Fig. 2. The probability of the leading particles production as a function of medium length $L$.

We have then studied the $\mathrm{L}$ dependence of the medium modification factor $I_{A A}$ (Fig. 3) by selecting different $x_{E}$ regions. For large $x_{E}$ particles, an obvious suppression is observed, and the suppression is stronger with increasing the medium length. For small $x_{E}$, the opposite behavior is obtained as an enhancement $\left(I_{A A}>1\right)$. This result implies that $\gamma$ hadrons correlation could be used to probe volume versus surface emission by selecting $\gamma$-jet events with different $x_{E}$ values. However such L dependence will be challenging to measure in the experiments.

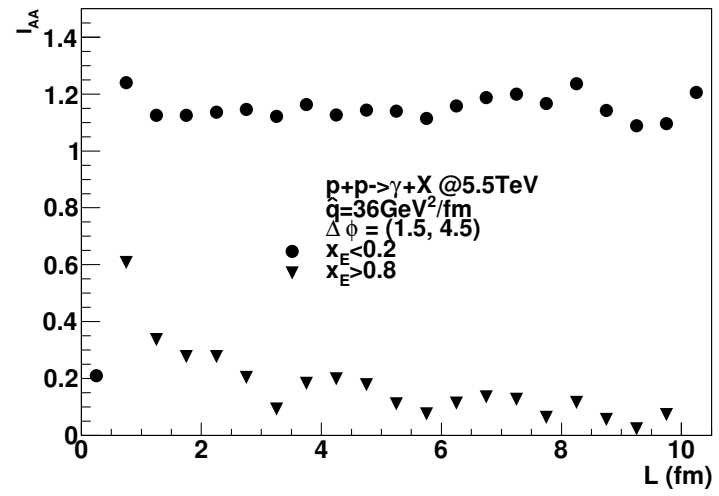

Fig. 3. The nuclear modification factor $I_{A A}$ distribution as a function of medium length $L$ by selecting different regions of $x_{E}$ on correlation distribution.

\section{Conclusions}

$\gamma+$ jet studies are widely recognized as a powerfull tool to characterize QGP. The " $\gamma+$ jet tomography" study will enable us to extract jet quenching parameters in different regions of the dense medium via measurement of the nuclear modification factor of $\gamma$-hadrons correlation.

We especially thank Prof.Xin-Nian Wang, Prof.Andreas Morsh, Prof.Peter Jacobs and Dr.Yuri Kharlov for their enthustic and fruitful discussions, also the full PWG4 workgroup in ALICE collabration.

\section{References}

1 http://www.bnl.gov/rhic

2 http://aliceinfo.cern.ch/Collaboration/

3 R. J. Fries and B. Muller, Eur. Phys. J. C 34 (2004) S279

4 H. Zhang, J. F. Owens, E. Wang and X. N. Wang, nuclth/0902.4000v1.

5 G. Conesa, et al., Nucl. Instr. and Meth. A 585 (2008) 28

6 Y. X. Mao, et al., Eur. Phys. J. C 57 (2008) 316-319.

7 M. Della Negra et al., Nucl. Phys. B 127 (1977) 1.

8 C. A. Salgado and U. A. Wiedemann, Phys. Rev. D 68 (2003) 014008.

9 T. Sjostrand et al., JHEP 0605 (2006) 026, hep-ph/0603175 (2006).

10 S. S. Adler, et al., Phys. Rev. D 74 (2006) 072002.

11 N. Armesto, G. Corcella, L. Cunqueiro and C. A. Salgado, hep-ph/0906.0754; hep-ph/0907.1014.

12 X. N. Wang, et al., Phys. Rev. C 55 (1997) 3047.

13 R. J. Glauber and G. Matthiae, Nucl. Phys. B 21 (1970) 135 .

14 M. Gyulassy and X. N. Wang, nucl-th/9502021 v1 (1995).

15 Y. X. Mao, et al., Chinese Physics C 32 (2008) 07. 